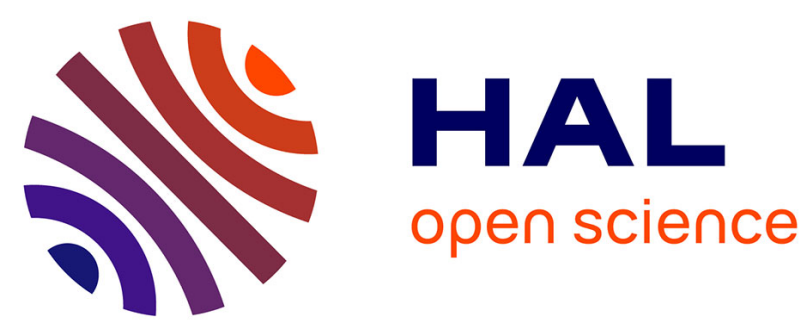

\title{
Performances of Low Rank Detectors Based on Random Matrix Theory with Application to STAP
}

Alice Combernoux, Frédéric Pascal, Marc Lesturgie, Guillaume Ginolhac

\section{To cite this version:}

Alice Combernoux, Frédéric Pascal, Marc Lesturgie, Guillaume Ginolhac. Performances of Low Rank Detectors Based on Random Matrix Theory with Application to STAP. 2014 International Radar Conference, Oct 2014, Lille, France. 10.1109/RADAR.2014.7060457 . hal-01084420

\section{HAL Id: hal-01084420 https://hal.science/hal-01084420}

Submitted on 19 Nov 2014

HAL is a multi-disciplinary open access archive for the deposit and dissemination of scientific research documents, whether they are published or not. The documents may come from teaching and research institutions in France or abroad, or from public or private research centers.
L'archive ouverte pluridisciplinaire HAL, est destinée au dépôt et à la diffusion de documents scientifiques de niveau recherche, publiés ou non, émanant des établissements d'enseignement et de recherche français ou étrangers, des laboratoires publics ou privés. 


\section{Performances of Low Rank Detectors Based on Random Matrix Theory with Application to STAP}

\author{
Alice Combernoux, Frédéric Pascal, \\ Marc Lesturgie \\ SONDRA - Supelec \\ Plateau du Moulon, 3 rue Joliot-Curie, \\ F-91192 Gif-sur-Yvette Cedex, France \\ Email: alice.combernoux@ @upelec.fr, frederic.pascal@ supelec.fr, \\ marc.lesturgie@supelec.fr
}

\author{
Guillaume Ginolhac \\ LISTIC - Polytech Annecy-Chambéry \\ 5 chemin de Bellevue, \\ 74944 Annecy-le-Vieux Cedex, France \\ Email: guillaume.ginolhac@univ-savoie.fr
}

\begin{abstract}
The paper addresses the problem of target detection embedded in a disturbance composed of a low rank Gaussian clutter and a white Gaussian noise. In this context, it is interesting to use an adaptive version of the Low Rank Normalized Matched Filter detector, denoted LR-ANMF, which is a function of the estimation of the projector onto the clutter subspace. In this paper, we show that the LR-ANMF detector based on the sample covariance matrix is consistent when the number of secondary data $K$ tends to infinity for a fixed data dimension $m$ but not consistent when $m$ and $K$ both tend to infinity at the same rate, i.e. $m / K \rightarrow c \in(0,1]$. Using the results of random matrix theory, we then propose a new version of the LR-ANMF which is consistent in both cases and compare it to a previous version, the LR-GSCM detector. The application of the detectors from random matrix theory on STAP (Space Time Adaptive Processing) data shows the interest of our approach.
\end{abstract}

Keywords-Low rank detection, Random matrix theory, GMUSIC estimator, Spike-MUSIC estimator, STAP processing, Adaptive Normalized Matched Filter.

\section{INTRODUCTION}

In the context of target detection in a noise composed of a low rank Gaussian clutter and an AWGN (Additive White Gaussian Noise), one could use the Low Rank Normalized Matched Filter (LR-NMF) detector [1] in order to exploit this low rank structure. Although its full rank version (NMF detector [2]) depends on the covariance matrix, the LR-NMF detector only requires the projector onto the clutter subspace. In practice, this projector and the covariance matrix are unknown and it is necessary to estimate them using $K$ secondary data which share the same properties as the tested data. It is well known that the adaptive low rank version of the detector needs much less secondary data as its classical version (ANMF detector [3]) for equivalent performances [4], [5].

Nevertheless, for high dimensional data, the performances of the LR-ANMF detector can suffer of this reality. Furthermore, the LR-ANMF detector is composed of 3 quadratic forms and, relying on [6] or [7], it is known that, although these quadratic forms are consistent when the number of secondary data $K$ tends to infinity for a fixed length $m$ of the data vector, they are no more consistent when the data length $m$ also tends to infinity. Hence it is interesting to use random matrix theory in order to provide consistent estimators of quadratic forms and more particularly of the LR-NMF detector which has been proved consistent when $K \rightarrow \infty$ with a fixed $m$ but no more consistent when $m, K \rightarrow \infty$ at the same rate, i.e. $m / K \rightarrow c \in(0,1]$. In this paper, we compare two versions of consistent detectors from random matrix theory and the Girko's estimators [8]. The first one, the LR-GSCM detector built from the G-MUSIC estimator [6], was presented and its convergence was studied in [9]. However the data model associated to this estimator is not suited to our data. Thus, we propose to develop another detector coming from the Spike-MUSIC estimator [7] which considers a data model closer to our data and which is designed to be consistent when the number of secondary data $K$ and the data dimension $m$ both tend to infinity at the same rate. In this paper, we study the consistency of the proposed detector. In addition, we compare the LR-GSCM detector and the proposed detector in terms of MSE and of detection performances through false alarm probability and detection probability.

As an illustration of the interest of the proposed detector, the STAP application [10] is studied as it is well known that the disturbance is then composed of a low rank Gaussian clutter plus a white Gaussian noise. Moreover, the dimension of the STAP data is often large with respect to the available number of secondary data.

The paper is organized as follows: Section II presents the problem statement and the definition of the LR-NMF detector. Section III contains the presentation of the studied detectors. Finally, Section IV shows the STAP application which illustrates the obtained results.

Notations: An italic letter stands for a scalar quantity, boldface lowercase (uppercase) characters stand for vectors (matrices) and (.) ${ }^{H}$ stands for the conjugate transpose. $\mathbf{I}_{N}$ is the $N \times N$ identity matrix. $\operatorname{diag}($.$) denotes the diago-$ nalization operator such as $(\mathbf{A})_{i, i}=(\operatorname{diag}(\mathbf{a}))_{i, i}=(\mathbf{a})_{i, i}$ and equal to zero otherwise. $\llbracket a, b \rrbracket$ is the set defined by $\left\{x \in \mathbb{Z}: a \leqslant x \leqslant b, \forall(a, b) \in \mathbb{Z}^{2}\right\}$.

\section{LOW RANK (LR) DETECTION}

\section{A. Problem formulation}

The aim of the problem is to detect a complex signal $\boldsymbol{d}$ in an additive noise $\boldsymbol{c}+\boldsymbol{n}$ in the observation vector $\boldsymbol{x} \in \mathbb{C}^{m \times 1}$. 
Hence, one can define the detection problem with the following binary hypothesis test:

$$
\left\{\begin{array}{lll}
H_{0}: \boldsymbol{x}=\boldsymbol{c}+\boldsymbol{n} & \boldsymbol{x}_{k}=\boldsymbol{c}_{k}+\boldsymbol{n}_{k}, & k \in \llbracket 1, K \rrbracket \\
H_{1}: \boldsymbol{x}=\boldsymbol{d}+\boldsymbol{c}+\boldsymbol{n} & \boldsymbol{x}_{k}=\boldsymbol{c}_{k}+\boldsymbol{n}_{k}, & k \in \llbracket 1, K \rrbracket
\end{array}\right.
$$

where $K$ is the number of secondary data, $\boldsymbol{x}_{k} \in \mathbb{C}^{m \times 1}$ are the secondary data (learning data used for the estimation of the total noise covariance matrix) and $\boldsymbol{n} \in \mathbb{C}^{m \times 1}$ (or $\boldsymbol{n}_{k}$ ) $\sim \mathcal{C N}\left(0, \sigma^{2} \mathbf{I}_{m}\right)$ is the AWGN (Additive White Gaussian Noise) complex vector. $\boldsymbol{d}$ is the target response and is equal to $\alpha \boldsymbol{a}(\boldsymbol{\Theta})$ where $\alpha$ is an unknown deterministic parameter, $\boldsymbol{a}(\boldsymbol{\Theta})$ is the steering vector and $\Theta$ is an unknown deterministic vector containing the localization parameters of the target. The clutter $c \in \mathbb{C}^{m \times 1}$ is modeled by a random centered complex Gaussian vector with a covariance matrix $\mathbf{C}\left(\boldsymbol{c}\right.$ or $\boldsymbol{c}_{k} \sim \mathcal{C N}(\mathbf{0}, \mathbf{C})$ ). The covariance matrix is normalized as $\operatorname{tr}(\mathbf{C})=m$. Consequently, the covariance matrix of the secondary data can be written as $\mathbf{R}=\mathbf{C}+\sigma^{2} \mathbf{I}_{m} \in \mathbb{C}^{m \times m}$. Moreover, the clutter is considered of low rank $r$ (as in a STAP application according to Brennan's formula [11]). Hence, $\operatorname{rank}(\mathbf{C})=r \ll m$ and one could write the eigendecomposition of $\mathbf{C}$ and define:

$$
\mathbf{C}=\sum_{i=1}^{r} \gamma_{i} \boldsymbol{u}_{i} \boldsymbol{u}_{i}^{H}
$$

where $\gamma_{i}$ and $\boldsymbol{u}_{i}, i \in \llbracket 1 ; r \rrbracket$ are respectively the non zero eigenvalues and the associated eigenvectors of $\mathbf{C}$, unknown in practice. The covariance matrix $\mathbf{R}$ of the secondary data can be decomposed as:

$$
\mathbf{R}=\sum_{i=1}^{m} \lambda_{i} \boldsymbol{u}_{i} \boldsymbol{u}_{i}^{H}
$$

with $\lambda_{1}=\gamma_{1}+\sigma^{2}>\cdots>\lambda_{r}=\gamma_{r}+\sigma^{2}>\lambda_{r+1}=$ $\cdots=\lambda_{m}=\sigma^{2}$. Then, we define the projector onto the clutter subspace $\Pi_{\mathrm{c}}$ and the projector onto the orthogonal subspace to the clutter subspace $\Pi_{\mathrm{c}}^{\perp}$ :

$$
\begin{aligned}
& \boldsymbol{\Pi}_{\mathrm{c}}=\sum_{i=1}^{r} \boldsymbol{u}_{i} \boldsymbol{u}_{i}^{H} \\
& \boldsymbol{\Pi}_{\mathrm{c}}^{\perp}=\mathbf{I}_{m}-\boldsymbol{\Pi}_{\mathrm{c}}=\sum_{i=r+1}^{m} \boldsymbol{u}_{i} \boldsymbol{u}_{i}^{H}
\end{aligned}
$$

\section{B. LR-NMF detector}

A filtering preprocessing on the observation vector $\boldsymbol{x}$ is first done in order to remove the clutter, and we retrieve a complex signal detection problem defined by the following binary hypothesis test:

$$
\left\{\begin{array}{l}
H_{0}: \boldsymbol{r}=\mathbf{U}_{0}^{H} \boldsymbol{x}=\boldsymbol{n}_{0} \\
H_{1}: \boldsymbol{r}=\mathbf{U}_{0}^{H} \boldsymbol{x}=\boldsymbol{d}_{0}+\boldsymbol{n}_{0}
\end{array}\right.
$$

where $\mathbf{U}_{0}=\left[\boldsymbol{u}_{r+1}, \cdots, \boldsymbol{u}_{m}\right]$. The detection problem is solved considering the white noise power $\boldsymbol{n}_{0}$ unknown. The used detection test corresponds to the Normalized Matched Filter in its low rank version, denoted by LR-NMF (Low Rank Normalized Matched Filter [1]):

$$
\begin{aligned}
& \Lambda_{\mathrm{LR}-\mathrm{NMF}}(\boldsymbol{\Theta})= \\
& \frac{\left|\boldsymbol{a}(\boldsymbol{\Theta})^{H} \boldsymbol{\Pi}_{\mathrm{c}}^{\perp} \boldsymbol{x}\right|^{2}}{\left(\boldsymbol{a}(\boldsymbol{\Theta})^{H} \boldsymbol{\Pi}_{\mathrm{c}}^{\perp} \boldsymbol{a}(\boldsymbol{\Theta})\right)\left(\boldsymbol{x}^{H} \boldsymbol{\Pi}_{\mathrm{c}}^{\perp} \boldsymbol{x}\right)} \underset{H_{0}}{\stackrel{H_{1}}{\gtrless}} \delta_{\mathrm{NMF}}
\end{aligned}
$$

where $\underset{H_{0}}{\stackrel{H_{1}}{\gtrless}} \delta_{\mathrm{NMF}}$ means that the $H_{1}$ hypothesis (respectively $\left.H_{0}\right)$ is decided if the test $\Lambda_{\mathrm{LR}-\mathrm{NMF}}(\boldsymbol{\Theta})$ is over (respectively under) the threshold $\delta_{\mathrm{NMF}}$.

\section{Regulation}

Observing Eq.(6), one can remark that if the steering vector is in the clutter, the left part of the denominator $\left(\boldsymbol{a}(\boldsymbol{\Theta})^{H} \boldsymbol{\Pi}_{\mathrm{c}}^{\perp} \boldsymbol{a}(\boldsymbol{\Theta})\right)$ is equal to zero and leads to an undefined value of the detector. Consequently, divergence problems will occur due to this division. Hence, we propose to regulate this part of the denominator according to the following equation:

$$
\begin{aligned}
& \Lambda_{\mathrm{LR}-\mathrm{NMF}}(\boldsymbol{\Theta})= \\
& \frac{\left|\boldsymbol{a}(\boldsymbol{\Theta})^{H} \boldsymbol{\Pi}_{\mathrm{c}}^{\perp} \boldsymbol{x}\right|^{2}}{\left(\boldsymbol{a}(\boldsymbol{\Theta})^{H} \boldsymbol{\Pi}_{\mathrm{c}}^{\perp} \boldsymbol{a}(\boldsymbol{\Theta})+\varepsilon\right)\left(\boldsymbol{x}^{H} \boldsymbol{\Pi}_{\mathrm{c}}^{\perp} \boldsymbol{x}\right)} \underset{H_{0}}{\stackrel{H_{1}}{\gtrless}} \delta_{\mathrm{NMF}}
\end{aligned}
$$

where $\varepsilon$ is the regulation parameter. In the remainder of the paper, the detectors will be used in their regulated version.

\section{ADAPTIVE LOW RANK DETECTORS}

\section{A. Traditional low rank detector}

The traditional estimation of the total noise covariance matrix $\mathbf{R}$ and the projector $\boldsymbol{\Pi}_{\mathrm{c}}^{\perp}$ orthogonal to the clutter subspace are first presented as, in practice, they are unknown. The estimation is based on the Sample Covariance Matrix (SCM) which is computed from the $K$ secondary data and can be written as:

$$
\hat{\mathbf{R}}_{\mathrm{SCM}}=\frac{1}{K} \sum_{k=1}^{K} \boldsymbol{x}_{k} \boldsymbol{x}_{k}^{H}=\sum_{i=1}^{m} \hat{\lambda}_{i} \hat{\boldsymbol{u}}_{i} \hat{\boldsymbol{u}}_{i}^{H}
$$

where $\hat{\lambda}_{i}$ and $\hat{\boldsymbol{u}}_{i}$ are respectively the eigenvalues and the eigenvectors. Finally, the estimated projectors are:

$$
\begin{aligned}
\hat{\boldsymbol{\Pi}}_{\mathrm{c}, \mathrm{SCM}} & =\sum_{i=1}^{r} \hat{\boldsymbol{u}}_{i} \hat{\boldsymbol{u}}_{i}^{H} \\
\hat{\boldsymbol{\Pi}}_{\mathrm{c}, \mathrm{SCM}}^{\perp} & =\mathbf{I}_{m}-\hat{\boldsymbol{\Pi}}_{\mathrm{c}, \mathrm{SCM}}=\sum_{i=r+1}^{m} \hat{\boldsymbol{u}}_{i} \hat{\boldsymbol{u}}_{i}^{H}
\end{aligned}
$$

Then, using the SCM, the estimated LR-SCM detector can be written as:

$$
\begin{aligned}
& \hat{\Lambda}_{\mathrm{LR}-\mathrm{SCM}}(\boldsymbol{\Theta})= \\
& \left.\Lambda_{\mathrm{LR}-\mathrm{NMF}}(\boldsymbol{\Theta})\right|_{\boldsymbol{\Pi}_{\mathrm{c}}^{\perp}=\hat{\boldsymbol{\Pi}}_{\mathrm{c}, \mathrm{SCM}}^{\perp}} \underset{H_{0}}{\stackrel{H_{1}}{\gtrless}} \delta_{\mathrm{SCM}}
\end{aligned}
$$

Although this detector is consistent when $K \rightarrow \infty$, it has been proved [9] that it is inconsistent when both $m, K \rightarrow \infty$ at the same rate $c=m / K$. That is why we use random matrix theory which permits to give us consistent estimators at this convergence regime under some assumptions.

\section{B. Low rank detectors from random matrix theory}

We first consider data without a priori on them: i.e. the secondary data without target can be written as $\boldsymbol{x}_{k}=\mathbf{R}^{1 / 2} \boldsymbol{y}_{k}$ where the elements of $\boldsymbol{y}_{k}, k \in \llbracket 1, K \rrbracket$, are i.i.d. and of p.d.f. $\mathcal{C N}(0,1)$ without any structure of covariance matrix $\mathbf{R}$. The corresponding estimator in random matrix theory is then the G-MUSIC estimator [6] and a consistent detector estimator was proposed [9] named LR-GSCM, based on this estimator:

$$
\begin{aligned}
& \hat{\Lambda}_{\mathrm{LR}-\mathrm{GSCM}}(\boldsymbol{\Theta})= \\
& \left.\Lambda_{\mathrm{LR}-\mathrm{NMF}}(\boldsymbol{\Theta})\right|_{\Pi_{\mathrm{c}}^{\perp}=\hat{\Pi}_{\mathrm{c}, \mathrm{GSCM}}^{\perp}} \underset{H_{0}}{\stackrel{H_{1}}{\gtrless}} \delta_{\mathrm{GSCM}}
\end{aligned}
$$


where $\hat{\boldsymbol{\Pi}}_{\mathrm{c}, \mathrm{GSCM}}^{\perp}=\sum_{i=1}^{m} \phi(i) \hat{\boldsymbol{u}}_{i} \hat{\boldsymbol{u}}_{i}^{H}$, with:

$$
\phi(i)=\left\{\begin{array}{l}
1+\sum_{n=1}^{r}\left(\frac{\hat{\lambda}_{n}}{\hat{\lambda}_{i}-\hat{\lambda}_{n}}-\frac{\hat{\mu}_{n}}{\hat{\lambda}_{i}-\hat{\mu}_{n}}\right), \text { if } i>r \\
-\sum_{n=r+1}^{m}\left(\frac{\hat{\lambda}_{n}}{\hat{\lambda}_{i}-\hat{\lambda}_{n}}-\frac{\hat{\mu}_{n}}{\hat{\lambda}_{i}-\hat{\mu}_{n}}\right), \text { if } i \leqslant r
\end{array}\right.
$$

where $\hat{\mu}_{1} \geqslant \cdots \geqslant \hat{\mu}_{m}$ are the eigenvalues of $\operatorname{diag}(\hat{\boldsymbol{\lambda}})-$ $\frac{1}{K} \sqrt{\hat{\lambda}} \sqrt{\hat{\lambda}}$ and $\hat{\lambda}=\left[\hat{\lambda}_{1}, \cdots, \hat{\lambda}_{m}\right]^{T}$. When $c>1, \hat{\mu}_{m}=$ $\cdots=\hat{\mu}_{K}=0$ and if $c=1, \hat{\mu}_{m}=0$.

However, this estimator does not really correspond to our problem due to the data model. Indeed, it does not bring any information about the data. In our problem one deals with a low rank Gaussian noise with a white Gaussian noise, which corresponds to the spiked model in random matrix theory presented by the authors of [7]. They consider that the secondary data can be written as:

$$
\boldsymbol{x}_{k}=\boldsymbol{c}_{k}+\boldsymbol{n}_{k}=\mathbf{R}^{1 / 2} \boldsymbol{y}_{k}=\left(\mathbf{C}+\sigma^{2} \mathbf{I}_{m}\right)^{1 / 2} \boldsymbol{y}_{k}
$$

where the elements of $\boldsymbol{y}_{k}$ are i.i.d. and of p.d.f. $\mathcal{C N}(0,1)$ and $\mathbf{C}$ is a rank $r$ perturbation matrix. Thus, the main difference between these two models (the first presented model and the spiked model) is the probability density function of the eigenvalues of the SCM (see [12] for the first presented model and [13] for the spiked model). Then they defined a consistent estimator of the quadratic form $\mathbf{s}_{1}^{H} \boldsymbol{\Pi}_{\mathrm{c}}^{\perp} \mathbf{s}_{2}$ derived from the data model:

$$
\mathbf{s}_{1}^{H} \hat{\boldsymbol{\Pi}}_{\mathrm{c}, \mathrm{SPIKE}}^{\perp} \mathbf{S}_{2} \underset{m, K \rightarrow \infty, m / K \rightarrow c}{\text { a.s. }} \mathbf{s}_{1}^{H} \boldsymbol{\Pi}_{\mathrm{c}}^{\perp} \mathbf{s}_{2}
$$

where $\hat{\boldsymbol{\Pi}}_{\mathrm{c}, \mathrm{SPIKE}}^{\perp}$ is a pseudo-projector taking into account all the estimated eigenvectors from the SCM and leading to a better estimation of the quadratic form. It can be written as $\hat{\boldsymbol{\Pi}}_{\mathrm{c}, \mathrm{SPIKE}}^{\perp}=\sum_{i=1}^{m} \xi(i) \hat{\boldsymbol{u}}_{i} \hat{\boldsymbol{u}}_{i}^{H}$ [7], with:

$$
\xi(i)= \begin{cases}1, & \text { if } i>r \\ 1-\frac{1+c \hat{\omega}_{i}^{-1}}{1-c \hat{\omega}_{i}^{-2}}, & \text { if } i \leqslant r\end{cases}
$$

with $\hat{\omega}_{i}=\frac{1}{2}\left(\hat{\lambda}_{i}-(c+1)+\sqrt{\left(c+1-\hat{\lambda}_{i}\right)^{2}-4 c}\right)$.

Consequently, we propose another consistent estimator more accurate for our data model, named LR-SPIKE, based on the Spike-MUSIC estimator:

$$
\begin{aligned}
& \hat{\Lambda}_{\mathrm{LR}-\mathrm{SPIKE}}(\boldsymbol{\Theta})= \\
& \left.\Lambda_{\mathrm{LR}-\mathrm{NMF}}(\boldsymbol{\Theta})\right|_{\boldsymbol{\Pi}_{\mathrm{c}}^{\perp}=\hat{\boldsymbol{\Pi}}_{\mathrm{c}, \mathrm{SPIKE}}^{\perp}} \underset{H_{0}}{\stackrel{H_{1}}{\gtrless}} \delta_{\mathrm{SPIKE}}
\end{aligned}
$$

The proof of the consistency of the LR-SPIKE detector is analog to the proof of the consistency of the LR-GSCM detector [9].

\section{STAP SIMULATIONS}

\section{A. Parameters}

As an illustration of the interest of the LR-SPIKE detector (from the random matrix theory), the STAP processing application is chosen. The purpose of STAP is to detect a moving target thanks to a uniform linear antenna composed of $N$ sensors receiving $M$ pulses. In this section, we choose $N=4$ and $M=16$ in order to have a large number for the data dimension $m=N M=64$. In STAP application, $\boldsymbol{\Theta}=(\theta, v)$ where $\theta$ is the DoA (Direction of Arrival) and $v$ the object relative speed. The target DoA and its relative velocity with respect to the airborne (radar platform) velocity are $\theta_{d}=0^{\circ}$ and $v_{d}=35 \mathrm{~m} . \mathrm{s}^{-1}$. According to Brennan's formula, the clutter rank is equal to $r=N+(M-1) \beta=19$, with $\beta=1$ in our configuration. Then, the signal wavelength is $l_{0}=0.667 \mathrm{~m}$, the AWGN power is $\sigma^{2}=1$, the signal to noise ratio is $S N R=18 \mathrm{~dB}$ and the clutter to noise ratio is $C N R=30 \mathrm{~dB}$. The regulation parameter is equal to $\varepsilon=0.1$.

\section{B. MSE}

First, we illustrate the estimation gain with the MSE between the LR-NMF detector and the estimated ones (LR-SCM, LRGSCM or LR-SPIKE) for $\theta=\theta_{d}$ and $v=v_{d}$ in Fig.1 and for a parameter set of the clutter in Fig. 2 as a function of the number of secondary data $K$. The MSE are here measured over 10,000 realizations. We note that the MSEs between the LR-NMF and the LR-GSCM or LR-SPIKE detectors are lower or almost equal than the MSEs between the LR-NMF and the LR-SCM detectors, for the majority of the $K$ values. In consequence, the LR-NMF detector is better estimated with the estimators based on random matrix theory on this range of $K$.

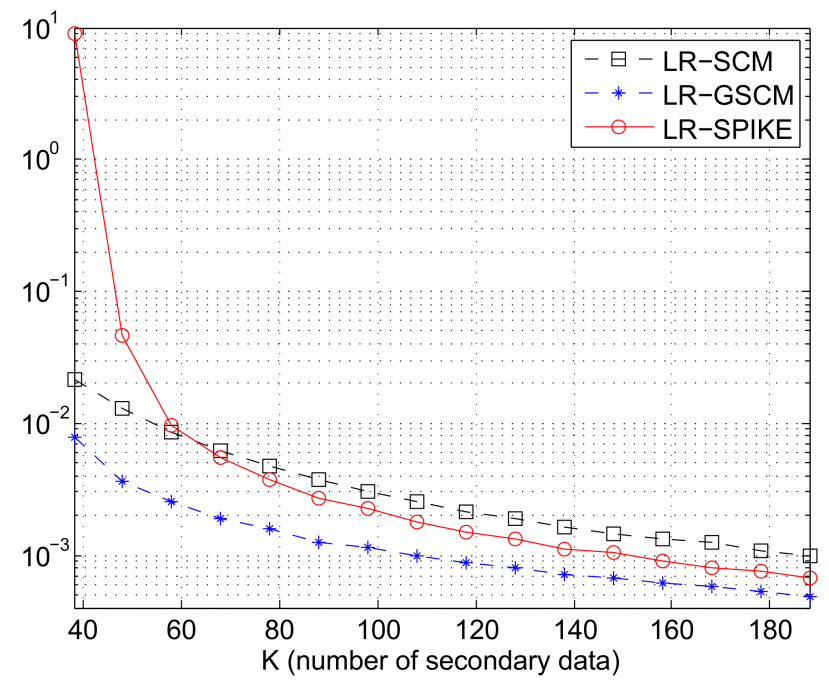

Fig. 1. MSE between the LR-NMF detector and the estimated ones (with regulation) at the target DoA and velocity.

\section{Pfa vs. threshold and ROC curves}

Then, the false alarm probability as a function of the threshold is shown in Fig. 3 and the detection probability as a function of the false alarm probability is shown in Fig. 4. We take here $K=m+1$. We first observe that the LR-GSCM detector leads to worst results that the classical LR-SCM detector due to the error of data model. However the LR-SPIKE detector leads to results identical to those of the classical LR-SCM detector.

At the end of this section, we can conclude that although the LR-SPIKE detector leads to results identical to those of the classical LR-SCM detector in terms of false alarm probability as a function of the threshold and detection probability as a function of the false alarm probability, the LR-SPIKE detector better estimate the response of the target. This gain is not noticeable on ROC curves nor Pfa vs. threshold as the MSEs 


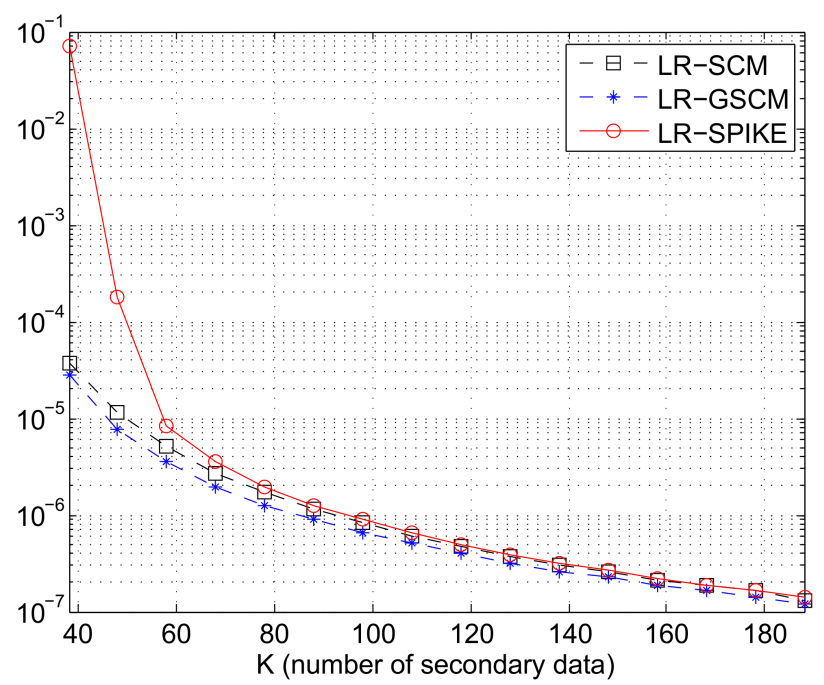

Fig. 2. MSE between the LR-NMF detector and the estimated ones (with regulation) at a parameter set of the clutter.

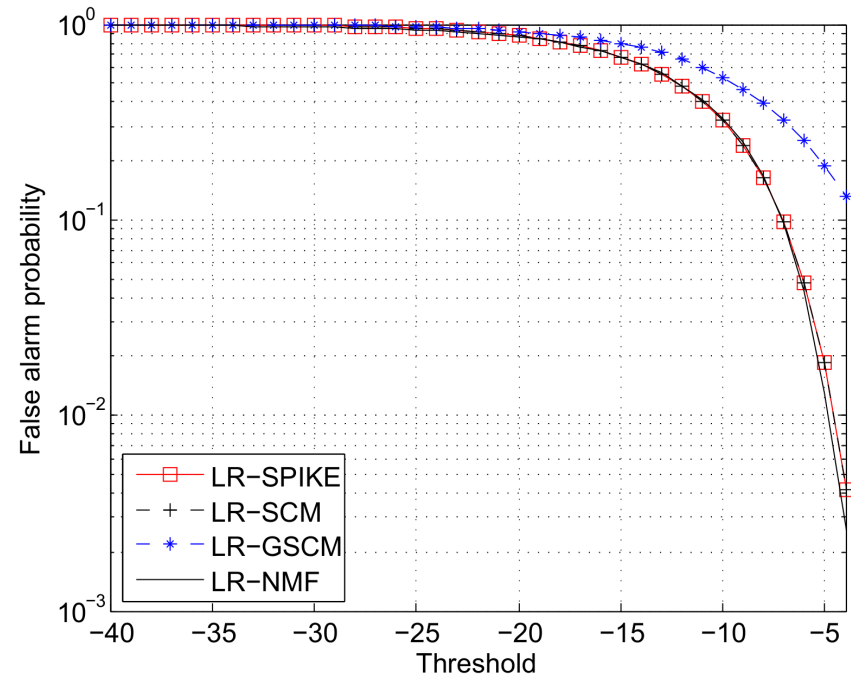

Fig. 3. False alarm probability as a function of the threshold. $\left(v_{d}=35 \mathrm{~m} \cdot \mathrm{s}^{-1}\right.$, $\theta_{d}=0^{\circ}, C N R=30 \mathrm{~dB}, S N R=18 \mathrm{~dB}$ and $\left.K=m+1\right)$

are computed when the noise is low compared to the target or the clutter. The LR-SPIKE detector is consequently more robust than the LR-GSCM detector.

\section{CONCLUSION}

In this paper we proposed a new adaptive low rank detector (LR-SPIKE) based on random matrix theory and more precisely on the Spike-MUSIC estimator. We studied its convergence when the number of secondary data $K$ and the data dimension $m$ both tend to infinity at the same rate and compared it to the LR-GSCM detector in terms of MSE vs. $K$ and in terms of detection probability vs. false alarm probability and false alarm probability vs. threshold in a STAP application. The reason of this interest is that the traditional low rank (LRSCM) detector, based on a simple eigendecomposition of the SCM is shown inconsistent when $K, m \rightarrow \infty$ at the same rate, contrary to the LR-GSCM and the LR-SPIKE detector. The results showed that the LR-SPIKE estimator, contrary to the LR-GSCM detector, gives similar results to the LR-

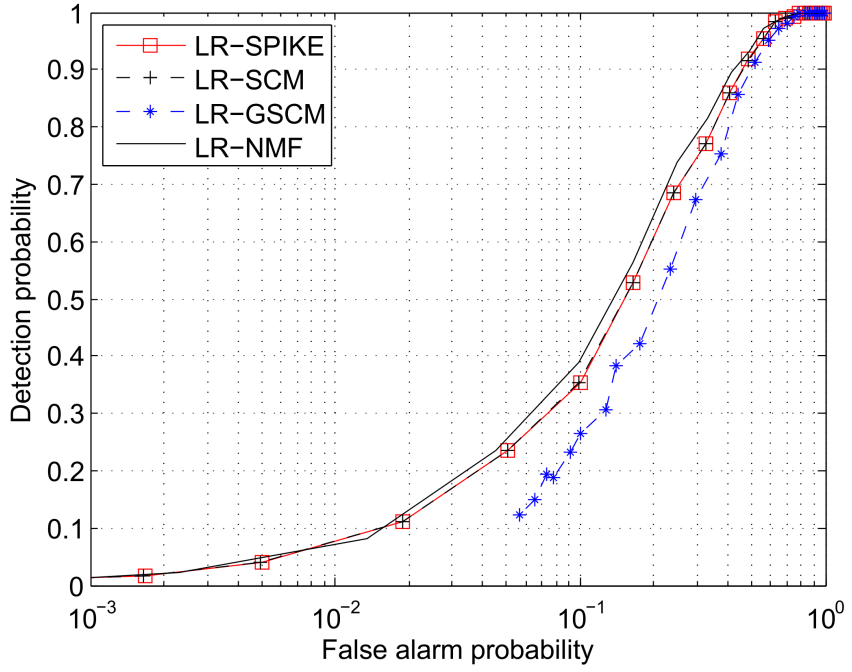

Fig. 4. Detection probability as a function of the false alarm probability. $\left(v_{d}=35 \mathrm{~m} \cdot \mathrm{s}^{-1}, \theta_{d}=0^{\circ}, C N R=30, S N R=18 \mathrm{~dB}\right.$ and $\left.K=m+1\right)$

SCM detector on ROC curves or Pfa vs. threshold but better estimates the LR-NMF detector for the majority of $K$. This gain is not noticeable on ROC curves nor Pfa vs. threshold as the MSEs are computed when the noise is low compared to the target or the clutter.

\section{REFERENCES}

[1] M. Rangaswamy, F. Lin, and K. Gerlach, "Robust adaptive signal processing methods for heterogeneous radar clutter scenarios," Signal Processing, vol. 84, pp. 1653 - 1665, 2004.

[2] L. Scharf and B. Friedlander, "Matched subspace detectors," IEEE Trans. on Sig. Proc., vol. 42, pp. 2146 - 2157, 1994.

[3] L. Scharf and L. M. Worther, "Adaptive matched subspace detector and adaptive coherence," Proc. of 30th Asilomar Conf. Signals, Syst., Comput., 1996.

[4] I. Kirstein and D. Tufts, "Adaptive detection using a low rank approximation to a data matrix," IEEE Trans. on Aero. and Elec. Syst., vol. 30, pp. $55-67,1994$

[5] A. Haimovich, "Asymptotic distribution of the conditional signal-tonoise ratio in an eigenanalysis-based adaptive array," IEEE Trans. on Aero. and Elec. Syst., vol. 33, pp. 988 - 997, 1997.

[6] X. Mestre and M. Lagunas, "Modified subspace algorithms for doa estimation with large arrays," IEEE Transactions on Information Theory, vol. 56, no. 2, pp. 598 - 614, February 2008.

[7] R. Couillet and W. Hachem, "Fluctuations of spiked random matrix models and failure diagnosis in sensor networks," IEEE Transactions on Information Theory, vol. 59, no. 1, pp. 509 - 525, 2013.

[8] V. Girko, An Introduction to Statistical Analysis of Random Arrays. VSP International Science Publishers, 1998, ch. 14 - Ten years of general statistical analysis, http://www.general-statisticalanalysis.girko.freewebspace.com/chapter14.pdf.

[9] A. Combernoux, F. Pascal, G. Ginolhac, and M. Lesturgie, "Random matrix theory applied to low rank stap detection," EUSIPCO, Sept. 2013, http://hal.archives-ouvertes.fr/hal-00871217.

[10] J. Ward, "Space-time adaptive processing for airborne radar," Lincoln Lab., MIT, Lexington, Mass., USA, Tech. Rep., December 1994.

[11] L. E. Brennan and F. Staudaher, "Subclutter visibility demonstration," RL-TR-92-21, Adaptive Sensors Incorporated, Tech. Rep., March 1992.

[12] X. Mestre, "Improved estimation of eigenvalues and eigenvectors of covariance matrices using their sample estimates," IEEE Transactions on Information Theory, vol. 54, no. 11, pp. 5113 - 5129, November 2008.

[13] J. Baik and J. W. Silverstein, "Eigenvalues of large sample covariance matrices of spiked population models," Journal of Multivariate Analysis, vol. 97 , pp. $1643-1697,2006$. 